\title{
Graft Copolymerization onto Chitosan V: Grafting Ethyl Acrylate onto Chitosan Initiated By Ceric Ion and Its Anti Microbial Activity
}

\author{
${ }^{1}$ Manoj Pati, ${ }^{2}$ P. L. Nayak \\ P.L Nayak Research Foundation, Eastern Academy of Science \& Technology, Odisha, India
}

\begin{abstract}
Ethyl Acrylate was grafted onto chitosan by using the ceric ammonium nitrate as the initiator. The effect of initiator concentration, monomer concentration, time \& temperature on $\% G$ and $\% E$ were studied. The grafted samples were characterized using FTIR, SEM, atomic force microscopy AFM and XRD methods. Xray diffraction showed changes in the crystallinity pattern of chitosan after the copolymerization reaction. Evidence of grafting was confirmed by FTIR spectroscopy. The morphology evaluated by SEM and AFM proves that in the absence of chitosan, latexes of vinyl homopolymers contain quasi monodispersed spheres with average diameter of about $450 \mathrm{~nm}$ or $250 \mathrm{~nm}$ while the graft copolymers latexes are formed by clustered irregular beads with average diameter less than $200 \mathrm{~nm}$. The antibacterial activities of the grafted polymer have also been investigated.
\end{abstract}

Keywords: Chitosan, Ethyl Acrylate, Graftcopolymerization, Antibactarial activity.

\section{Introduction}

Grafting vinyl monomers onto natural and synthetic polymers is a challenging field of research with unlimited future prospects to improve substantially the properties of the base polymer without sacrificing its properties During the last three decades, Prof Nayak and his group have extensively investigated graft copolymerization of vinyl monomers onto a large number of natural and synthetic polymers like wool, silk, cellulose, nylon, and PET, etc using a multitude of metal and non metal ion like hexavalent chromium, quinquevalent vanadium, tetravalent cerium, trivalent manganese, trivalent thalium, peroxydisulphate and peroxydiphosphate ions (1-14) as initiators. The greatest problem in the field of grafting is to use the exact initiator and reaction conditions to minimize the formation of homopolymer so that the properties of the base polymer could be enhanced. The homopolymer once formed on the back bone of the polymer is very difficult to remove from the base polymer by the usual solvent extraction technique

Chitosan is a linear polysaccharide derived from chitin, the second most abundant organic compounds only next to cellulose in the nature. Chitin can be found in the shells of marine invertebrates (crabs, crustaceans, etc.), fungi, insects and yeasts. Depending on the source, it generally functions as an exoskeleton, providing structural integrity, commonly embedded in a matrix of proteins, minerals and at times various other polysaccharides. Chitin is a homopolymer comprised of 2-acetamido-2-deoxy- $\beta$-D-glucopyranose units; however, some units exist in the deacetylated form as 2-amino-2-deoxy- $\beta$-D-glucopyranose. When chitin is deacetylated to at least $50 \%$, it is referred to as chitosan, in other words this is essentially the $\mathrm{N}$-deacetylated derivative of chitin (chemical structure is provided below in Figure 1A, where $\mathrm{x}=1-\mathrm{y}, \mathrm{y}$ being the proportion of acetylated repetition units).

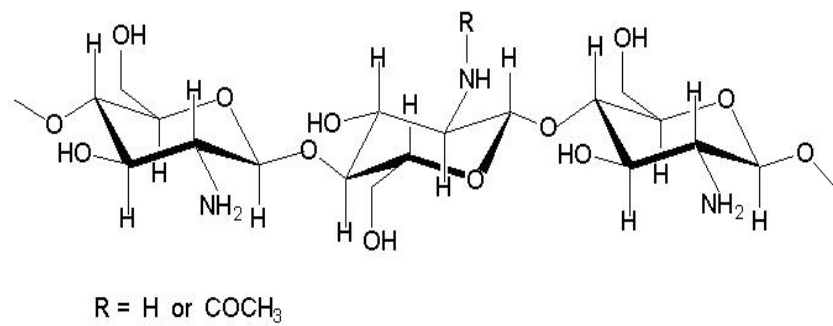

Fig-1 Structure of Chitosan

Chitosan has many interesting biological and chemical properties. The excellent features such as biocompatibility, ecologically safe biodegradability (degradation products of chitosan are nontoxic, nonimmunogenic and non-carcinogenic) and low toxicity with versatile biological activities (chitosan has antimicrobial activity and low immunogenicity) recommends this biopolymer for applications in biomedicine [15-20]. In addition, chitosan is very efficient for interaction with the anionic solutes including dyes in acidic 
Graft Copolymerization onto Chitosan V: Grafting Ethyl Acrylate onto Chitosan Initiated by Ceric solutions due to the reactive groups, such as $-\mathrm{OH}$ and $-\mathrm{NH} 2$. This property has been widely used for the removal of the water-soluble dyes, as an alternative to the conventional sorbents and flocculants [21, 22]. However, due to inter- and intra- molecular H-bonding, chitosan is only soluble in few dilute acid solutions (depending on the molecular weight), which limits its applications. As a result, many attempts have been made in chemical modification of chitosan, aiming at improving its water solubility. Among these an ideal way is the graft modification of chitosan [23-25]. The main advantage in the grafting efforts is the high degree of functionality of chitosan - the molecule backbone contains two hydroxyl groups and one primary amine group per repeat unit. The active primary amino groups on the molecule being reactive provide sites for the attachment of different side groups employing mild reaction conditions. In this way versatile materials based on chitosan with specific functionality can be obtained.

The antimicrobial property of chitosan and its derivatives, with conflicting results, has received considerable attention in recent years due to imminent problems associated with synthetic chemical agents. Such an application stems from the cationic charge of chitosan molecule to give rise to aggressive binding onto the microbial cell surface, leading to gradual shrinkage of cell membrane and finally death of the cell. Several possible explanations have been proposed for antimicrobial activity [26].It has been reported that quaternary ammonium salt of chitosan exhibits good antibacterial activities, for example, diethylmethylchitosan chloride showed higher antibacterial activity than chitosan. Novel N, O-acyl chitosan derivatives were more active against the gray mould fungus Botrytis cinerea and the rice leaf blast fungus Pyricularia oryzae; hydroxypropyl chitosan grafted with maleic acid sodium killed over $99 \%$ of Staphylococcus aureus and E. coli within 30 min of contact at a concentration of $100 \mathrm{ng} / \mathrm{ml}$; hydroxypropyl chitosan was a potent inhibitor of Azatobacter mali, Clostridium diplodiella, Fusarium oxysporum and Pyricularia piricola [27].

In the present research program, we wish to report the graft copolymerization of ethyl acrylate onto chitosan using ceric ammonium nitrate as the initiator. The graft co- polymrization was studied by varying the initiator, time, temperature and concentration of monomer. The grafted polymers were characterized by SEM, $\mathrm{XRD}$, AFM and FTIR studies. The antibacterial and antifungal activity of the grafted samples have been reported.

\section{Experimental}

Materials

Chitosan (CS) (Degree of Deacetylation $=95 \%$ determined by 1H-NMR and Molecular Weight 13.45 $\times 104 \mathrm{Da})$ was purchased from India Sea Foods, Kerela, India. Acetic acid, Ethyl Acrylate and other chemicals were used as analytical grade and purchased from Sigma Aldrich Company.

\section{Graft Copolymerization}

A chitosan aqueous solution of $2 \mathrm{wt} \%$ was prepared by dissolving $20 \mathrm{~g}$ of chitosan powder in $1000 \mathrm{~mL}$ of acetic acid solution $(1 \%, \mathrm{v} / \mathrm{v})$. After chitosan was dissolved, the solutions were filtered with cheesecloth by vacuum aspiration to remove foam and any undissolved impurities. The ceric ammonium nitrate in $0.5 \mathrm{M}$ nitric acid solution was then loaded into the reactor under continuous stirring. Then a known weight of ethyl acrylate was also injected into the reactor. The reaction was assumed to have started at the moment the monomer was injected. The grafting reaction was carried out under nitrogen atmosphere in a $500 \mathrm{~mL}$, four-necked flask equipped with a reflux condenser, a stirrer, dropping funnel, and a gas inlet system immersed in a constant temperature water bath. In a typical reaction, Chitosan $(0.006-0.025 \mathrm{~mol} ; 1-4 \mathrm{~g})$ was dispersed in a definite volume of water with constant stirring and bubbling of a slow stream of nitrogen for $30 \mathrm{~min}$ at the desired temperature $\left(20-40^{\circ} \mathrm{C}\right)$. After $30 \mathrm{~min}$, a freshly prepared $10 \mathrm{~mL}$ solution of CAN $(0.02-0.06 \mathrm{~mol}, 0.11-0.33 \mathrm{~g})$ in nitric acid $(0.1-0.4 \mathrm{~N})$ was added and stirred for $10 \mathrm{~min}$. Nitrogen gas was continuously passed through the reaction mixture and ethyl acrylate $(0.091-0.152 \mathrm{~mol}, 6-10 \mathrm{~mL})$ was added. In all the reactions, total volume of the reaction was kept constant. The grafting reaction was carried out for varying time intervals ( $1-4 \mathrm{~h})$. The zero time of the reaction was at the time of monomer addition. After completion of the reaction, the reaction mixture was immediately poured into methanol in the ratio of $1: 5$ of material to liquor for precipitation. The precipitated product was recovered by centrifugation and washed with pure methanol $(2 \times 50 \mathrm{~mL})$. The crude copolymer thus obtained was dried till constant weight under vacuum $(7.6 \mathrm{~mm} \mathrm{Hg})$ for $24 \mathrm{~h}$ at $40^{\circ} \mathrm{C}$. The dried product was extracted with dimethylformamide for $48 \mathrm{~h}$ and washed with methanol to remove the homopolymer (polyethyl acrylate ). The grafted Chitosan (Chitosan-g-ethyl acrylate) was dried to a constant weight under vacuum $(7.6 \mathrm{~mm} \mathrm{Hg})$ for $24 \mathrm{~h}$ at $40^{\circ} \mathrm{C}$. The percentage grafting $(\% \mathrm{G})$ and percentage grafting efficiency $(\% \mathrm{GE})$ were determined from the increase in the weight of Chitosan after grafting in the following manner:

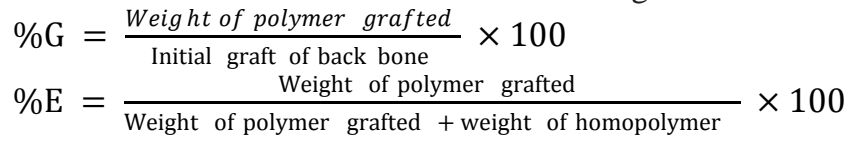



FTIR

The Fourier Transform Infrared Spectrum (FTIR) of grafted samples was measured in KBr pellets using a JASCO FTIR-5300 spectrophotometer in the range 4000-650 $\mathrm{cm}^{-1}$.

\section{Scanning electron microscopy studies}

Scanning electron microscopy images at 500 magnifications were obtained for Chitosan and Chitosang-ethyl acrylate using Zeiss EVO 40 EP Scanning Electron Microscope (Cambridge, England). The sample was laid on the aluminum stub using double-sided conducting adhesive tape and was sputter coated with gold.

\section{Atomic Force Microscopy (AFM)}

An Atomic Force Microscopy (AFM) with a MultiView 4000 Nanonics System, working in noncontact mode. For the SEM investigation the samples were sputtered with a thin layer of gold prior to imaging.

\section{XRD}

The X- ray diffraction studies were performed using a Philips-Holland diffractometer (model PW 1729 ) with copper as target material in an $\mathrm{X}$ - ray tube under the operational conditions $30 \mathrm{KV}, 40 \mathrm{~mA}$ and wavelength between 1.54060 and $1.54438 \mathrm{a}$. The samples were scanned between 3oand 100 .

\section{Antimicrobial Susceptibility Test}

The disc diffusion method was used to screen the antimicrobial activity. In vitro antimicrobial activity was screened by using Mueller Hinton Agar (MHA) obtained from Himedia (Mumbai). The MHA plates were pre- pared by pouring $15 \mathrm{ml}$ of molten media into sterile Petri plates. The plates were allowed to solidify for 5 min and $0.1 \%$ inoculum (0.5 McFarland standard) suspension was swabbed uniformly and the inoculum was allowed to dry for $5 \mathrm{~min} .50 \mu \mathrm{l}$ concentration of test sample was loaded on $0.5 \mathrm{~cm}$ sterile disc. The loaded disc was placed on the surface of medium and the compound was allowed to diffuse for $5 \mathrm{~min}$ and the plates were kept for incubation at $37^{\circ} \mathrm{C}$ for $24 \mathrm{~h}$. At the end of incubation, inhibition zones formed around the disc were measured with trans-parent ruler in millimeter. For each bacterial strain, negative controls were maintained where pure solvents were used instead of the extract. The control zones were subtracted from the test zones and the resulting zone diameter and the result obtained was tabulated) and Ampicillin $(10 \mathrm{mcg} / \mathrm{disc})$ were used.

\section{Result and Discussion}

CAN has been used extensively as the redox initiator for effecting grafting of a variety of vinyl monomers onto biopolymers. The formation of free radicals in $\mathrm{Ce}(\mathrm{IV})$-treated biopolymers has been confirmed by electron spin resonance. The mechanism by which $\mathrm{Ce}(\mathrm{IV})$ interacts with biopolymer to form free radical involves the formation of a coordination complex between the Ce(IV) and the hydroxyl group of biopolymer. The Ce(IV)-biopolymer complex then disproportionate forming a free radical on the biopolymer chain and $\mathrm{Ce}(\mathrm{III})$.

Evidence for complex formation has been obtained by kinetic and spectrophotometric methods for the oxidation of various alcohols and substrates containing alcohol groups by $\mathrm{Ce}(\mathrm{IV})$ ions in perchloric and nitric acids. The postulated mechanism has been supported by the model compound studies of $\mathrm{Ce}(\mathrm{IV})$ oxidation of monohydric alcohols and 1,2-glycols and suggest that the $\mathrm{C}_{2} \mathrm{AC}_{3}$ glycol and the $\mathrm{C}_{6}$ hydroxyl of an anhydro-Dglucose unit may be preferred sites for free-radical generation.

\section{Determination of the optimum reaction conditions}

To optimize the conditions for grafting of ethyl acrylate onto Chitosan, the concentration of nitric acid, free-radical initiator, monomer, Chitosan, time, and temperature were varied.

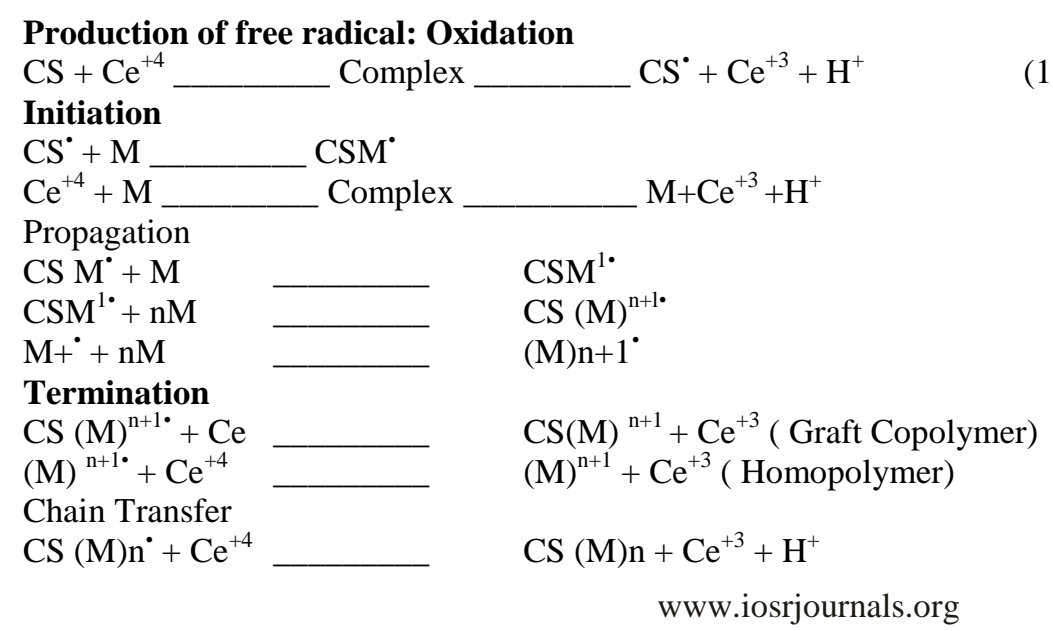

Initiation

$\mathrm{Ce}^{+4}+\mathrm{M}$ CSM

Propagation

$\mathrm{CS} \mathrm{M}+\mathrm{M}$

$\mathrm{CSM}^{1 \cdot}+\mathrm{nM}$

CS $(\mathrm{M})^{\mathrm{n}+1 \cdot}$

$\mathrm{M}+{ }^{\circ}+\mathrm{nM}$

Chain Transfer

3 |Page 
Graft Copolymerization onto Chitosan V: Grafting Ethyl Acrylate onto Chitosan Initiated by Ceric $\mathrm{CS}(\mathrm{M}) \mathrm{n}^{\circ}+\mathrm{M} \quad \mathrm{CS}(\mathrm{M}) \mathrm{n}+\mathrm{M}$.

(Where $\mathrm{CH}, \mathrm{m}, \mathrm{CH}(\mathrm{M})_{\mathrm{n}+1} \overline{\text { and }(\mathrm{M})_{\mathrm{n}+1}}$ represent chitosan, Ethyl acrylate, the graftcopolymer and homopolymer, respectively)

\section{Effect of CAN Concentration}

The effect of the concentration of the initiator $\mathrm{Ce}^{4+}$ on grafting of ethyl acrylate. It was observed that the maximum percentage of grafting occurred at $5.70 \times 10^{-3} \mathrm{M}$. A further increase in the $\mathrm{Ce}^{4+}$ concentration leads to a decrease in the grafting percentage of ethyl acrylate. This could be explained by the fact that ceric ion at a higher concentration causes the termination of grafting polymeric chain growth since ceric ion is a very good terminator. Another factor which could contribute to a decrease in the grafting percentage at higher concentration of initiator is the increase the homopolymer formation, which competes with the grafting reaction for the available monomer. The above things are explained by the figure-2 given as follows.

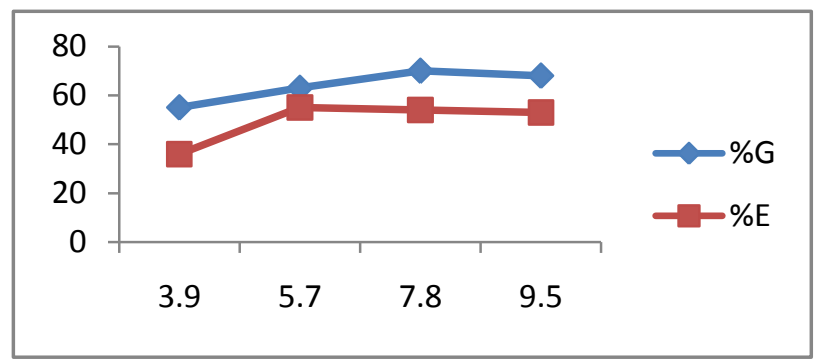

Fig-2 Effect of CAN Concentration

\section{Effect of monomer concentration}

The effect of the ethyl acrylate concentration on the graft yield obtained with chitosan is shown in figure-3. An increase in the monomer concentration is accompanied by significant increase in grafting up to $0.75 \mathrm{M}$. However with the further increase in the concentration of monomer, grafting is found to decreases. This decrease can be attributed to the higher affinity of ethyl acrylate monomer for its homopolymer over the chitosan macroradicals. Thus, most of the monomer is preferentially used up in the formation of homopolymer on increasing the ethyl acrylate concentration, which is evident from the rise in viscosity of the reaction medium at higher concentration.

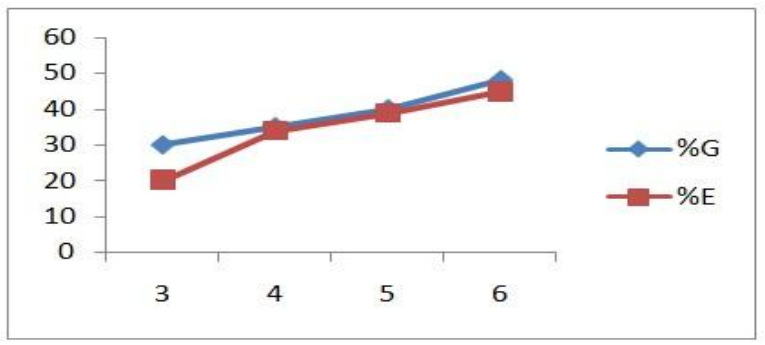

Fig-3 Effect of monomer concentration

\section{Effect of Time}

The effect of the reaction time on the percentage of grafting and grafting efficiency is shown in figure4. The percentage of grafting was found to increase linearly with time and then approximately constant. The initial increase in the rate due to the increase in the number of grafting site, but this number remain constant with further increase of time.

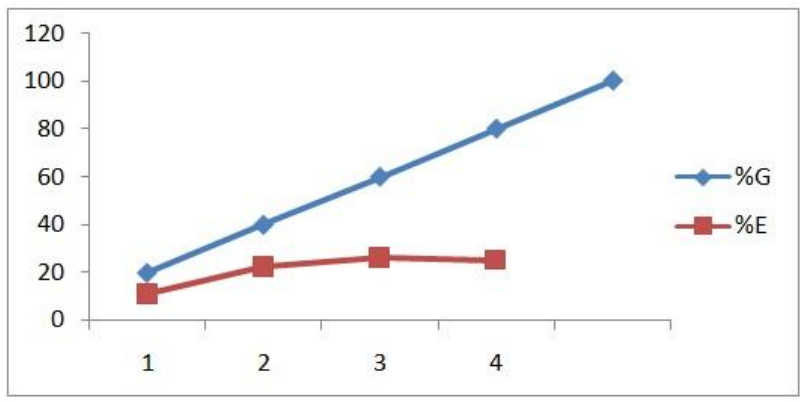

Fig-4 Effect of Time

www.iosrjournals.org 


\section{Effect of Temperature}

The dependence of grafting yield on temperature in the range of $35^{\circ} \mathrm{C}-55^{\circ} \mathrm{C}$ is shown in figure-5. The maximum grafting of ethyl acrylate occurs at $40^{\circ} \mathrm{C}$ within $180 \mathrm{~min}$. A further increase in temperature reduces the percentage grafting. This is to be expected since at higher temperature various chain transform reaction are accelerated which leads to a decrease in the percentage of grafting on in other words the formation of more homopolymer.

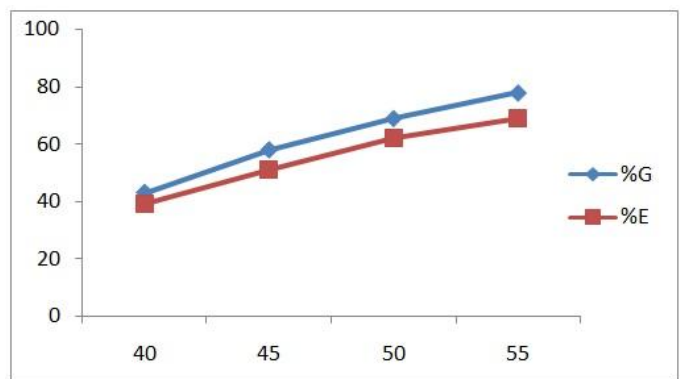

Fig-5 Effect of Temperature

\section{Scanning electron micrograph of Chitosan}

The scanning electron microscope picture of grafted chitosan is shown in the figure-6. It clearly exhibits the polysaccharide nature having varied particle sizes with rough surface. Even the polysaccharide seems completely scattered along with larger particles. A change in contour of the polysaccharide on grafting and the thick polymeric coating of ethyl acrylate on their surface along with grafting of ethyl acrylate such that all the gap between polysaccharide particles have been closed indicate the effect of grafting. It can be seen that individual polysaccharide molecules of Chitosan have joined through these surface coatings during grafting process.

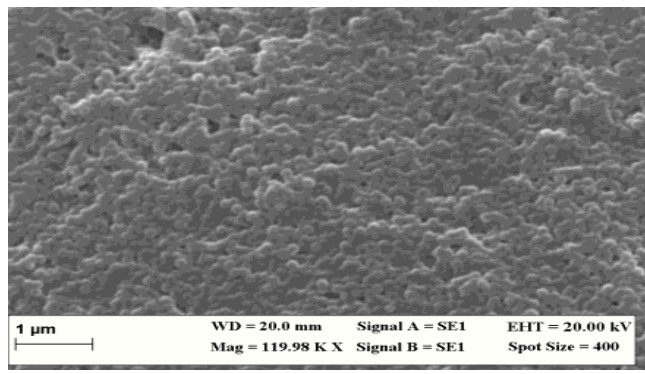

Fig-6 Scanning electron micrograph of grafted Chitosan

\section{X-ray diffraction studies}

In the first step the change of CS structure after graft copolymerization was investigated by X-ray diffraction. Figure 7 shows the XRD patterns of CS. CS shows distinct crystalline peak at around 20: 20.00; $30.00 ; 35.00$. The characteristic reflection at $2 \theta=20.00$ corresponds to the orthorhombic crystal structure of chitosan. The CS crystallinity is linked to the great number of $-\mathrm{OH}$ and $-\mathrm{NH} 2$ groups presented in its structure. These groups can form stronger inter- and intra- molecular H-bonds which lead to a certain regularity of the CS structure and have as result the appearance of crystalline regions. The graft copolymers XRD patterns CS-Ethtyl acylate exhibit broader and weaker peaks at $2 \theta=20.00$, implying that these materials were almost amorphous. Thus, the XRD result reveals that the ethyl acrylate chains can interfere with CS ordered packing, most probably by a steric effect which alters the H-bonding.

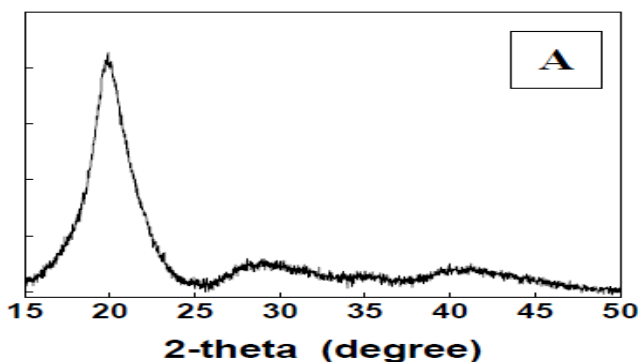

Fig-7 X-ray diffraction studies of CS/ethyl acrylate 

FTIR

The best tool to confirm the grafting reaction is infrared spectroscopy. Figure 8 shows the FTIR spectra of the graft copolymers in comparison with those of the homopolymers and parent chitosan. The FTIR spectrum of CS (Figure 8) showed features of amide groups: amide I, amide II and amide III bands at 1651, 1524 and $1319 \mathrm{~cm}-1$, respectively. The absorption band located at $1651 \mathrm{~cm}-1$ linked to the acetamide group $\left(-\mathrm{NHCOCH}_{3}\right)$ confirms the partial degree of deacetylation of CS. The intense band at $3370 \mathrm{~cm}-1$ is attributed to the overlapping of $\mathrm{O}-\mathrm{H}$ and $\mathrm{N}-\mathrm{H}$ stretching vibrations, as well as to intermolecular $\mathrm{H}$-bonds within the polysaccharide. The absorption bands at $1153 \mathrm{~cm}-1$ (anti-symmetric stretching of the $\mathrm{C}-\mathrm{O}-\mathrm{C}$ bridge), $1073 \mathrm{~cm}-$ 1 and $1038 \mathrm{~cm}-1$ (skeletal vibrations $\mathrm{C}-\mathrm{O}$ stretching) are characteristic absorption bands for chitosan as saccharide structure.
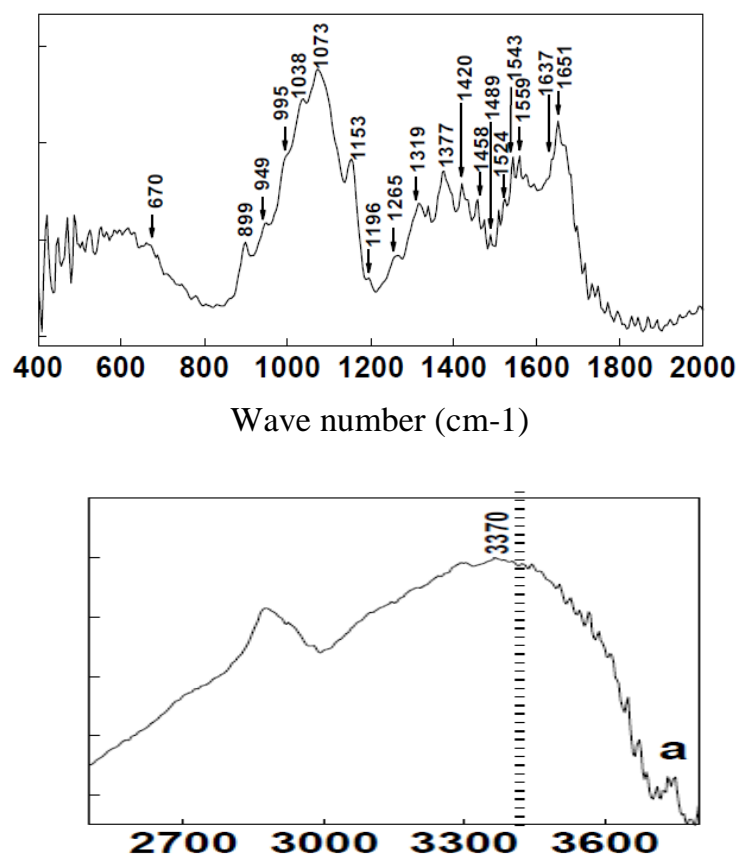

Wavenumber (cm-1)

Fig 8 FTIR of studies of CS/ethyl acrylate

\section{Air Force Microscope (AFM)}

The AFM report of grafted chitosan is shown in the figure 9. It gives the information which is based on the homopolymers latexes are formed by spherical particles having average diameter $\sim 450 \mathrm{~nm}$ and $\sim 250 \mathrm{~nm}$ also confirmed by AFM images. The optical images of graft copolymers: suggest an agglomeration process, this being emphasized mostly in the case of CS-ethyl acrylate sample leading to larger aggregates formation. The hypothesis is sustained by the AFM images.

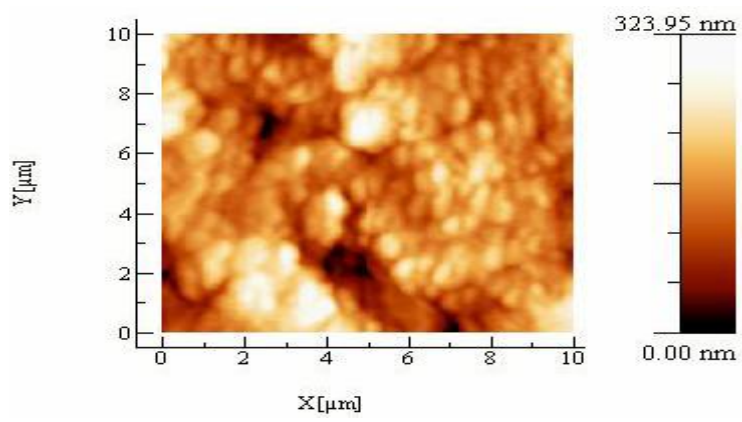

Fig 9 AFM of Chitosan-ethyl acrylate

\section{Antibacterial Activities of Grafting compound :}

A preliminary study has been carried out to compare the antibacterial activity of grafted chitosan hydrochloride film samples with that of chitosan. Grafted chitosan were efficient in inhibiting the against Escherichia coli, Klebsiella , B.cereus , E.coli ,Pseudomonas. Figure 10 is a photo of a Petri dish showing the zones of inhibition. The results are shown in Table 1. The highest zone of growth of inhibition occurs at a 
Graft Copolymerization onto Chitosan V: Grafting Ethyl Acrylate onto Chitosan Initiated by Ceric concentration of $100 \mathrm{~m} \%$, while the lowest zone of growth inhibition occurs at a concentration of $6.25 \%$. The antibacterial profile of essential grafting compound of chitosan-ethyl acrylate was studied. Like essential grafting compound was found to be effective against all the four bacteria B.cereus, E.coli, klebsiella, Pseudomonas. All the bacteria, tested were inhibited at $100 \%, 50 \%, 25 \%, 12.5 \& 6.25 \%$ concentration of essential grafting compound along with control. The result of zone of inhibition after $24 \mathrm{hr}$ was reported in (Table 1 ).The essential grafting sample was found to be sensitive against B.cereus. The minimum inhibitory concentration was found to be $25 \%$ while for essential grafting sample, minimum inhibitory concentration was found to be $12.5 \%$ (Table 1). The zone of inhibition of essential grafting sample for E.coli with control was $12.60 \mathrm{~mm}$. The essential grafting sample was found to be resistant to bacterium. The zone of inhibition for Pseudomonas with control was $11.45 \mathrm{~mm}$. The grafting sample was found to be sensitive against Pseudomonas. The minimum inhibitory concentration was found to be $25 \%$.

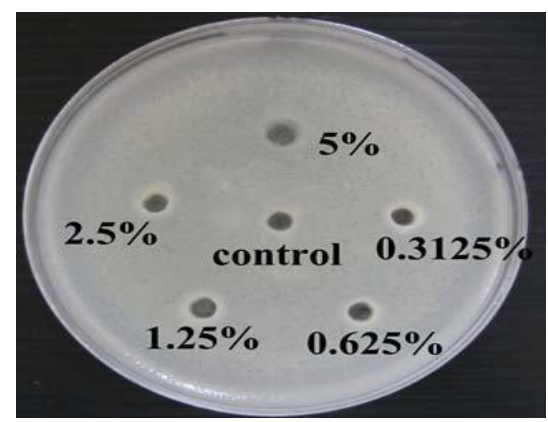

Fig 10 Anti bacterial Activities of Grafted chitosan

\begin{tabular}{|l|c|c|c|c|c|c|l|}
\hline \multirow{2}{*}{ Microorganism } & \multicolumn{5}{|c|}{$\begin{array}{c}\text { Inhibition zone (in mm) } \\
\text { Concentration }\end{array}$} & $\begin{array}{c}\text { Minima } \\
\text { Control } \\
\text { inhibitory } \\
\text { concentration } \\
\text { (mic \%) }\end{array}$ \\
\hline B.cereus & 10.73 & 9.68 & 8.82 & 6.43 & 6.22 & 10.46 & 25 \\
\hline E.coli & 10.23 & 9.45 & 8.32 & 6.24 & NZ* & 12.60 & resistance \\
\hline Klebsiella & 9.44 & 8.78 & 7.95 & NZ* & NZ* & 12.14 & resistance \\
\hline Pseudomonas & 11.26 & 10.48 & 9.45 & 7.64 & NZ* & 11.45 & 25 \\
\hline
\end{tabular}

Table-1 Antibactarial activities of Grafting CS/ethyl acrylate Compound minimal inhibitory concentration (MIC) $\mathrm{mg} / \mathrm{ml}$.

\section{Conclusion}

The results reported in this study provide a useful and simple synthesis route, surfactant-free emulsion copolymerization, for developing bead-like particles copolymers based on chitosan and ethyl acrylate . The graft copolymerization of ethyl acrylate onto Chitosan in aqueous medium was initiated effectively with CAN. The reaction conditions were optimized for grafting of ethyl acrylate onto Chitosan by varying the concentration of Chitosan, CAN, ethyl acrylate, $\mathrm{HNO}_{3}$, polymerization time, and reaction temperature. The samples was characterized by different techniques: X-ray diffraction, FTIR spectroscopy, SEM and AFM. X-ray diffraction patterns revealed changes in the crystallinity of grafted copolymers. The FTIR spectra confirmed the grafting reaction due to the presence of fingerprints absorption bands of the both components, . The microscopy images revealed that quasi-monodispersed sphere particles (homopolymes latexes) or clustered irregular beads (grafted copolymers latexes) are formed by the SFEP technique. It is expected that bead-particles based on chitosan and ethyl acrylate can be synthesized in order to obtain advance multifunctional materials for applications in biotechnological fields. This study has demonstrated that grafting compound showed a higher antibacterial activity. Grafting compound markedly inhibited the growth of most bacteria tested although their inhibitory effects differed.

\section{Acknowledgements}

Authors are grateful to the Director of CIPET, Bhubaneswar, Orissa for SEM, FTIR, XRD and AFM analysis and Manager of Bio-Lab (A-41, Janpath, Ashok Nagar, Bhubaneswar, India 751009). 


\section{Reference}

[1] Nayak P.L., J. Macromol. Sci-Rev. macromol. Che. C14 (2) (1976) 193.

[2] Nayak P.L. Lenka .S and Pati N.C J. Macromol. Angew, macromol. Che: 71; (1978) 189.

[3] Nayak P.L. Lenka .S and Pati N.C, J. Applied Poly. Science: 23; (1979) 1345.

[4] Nayak P.L.,Lenka .S and Pati N.C, J. of Applied Poly. Science.: 17; (1979) 3425.

[5] Nayak P.L., Lenka .S and Pati N.C, J. Macromol. Sci-Rev. macromol. Che.: A13; (1979) 1157

[6] Nayak P.L., Lenka .S and Pati N.C, J. Macromol. Sci-Rev. macromol. Chem.: 85; (1980) 29.

[7] Nayak P.L., Lenka .S and Pati N.C, J. Macromol. Sci-Rev. macromol. Chem.: 84; (1980) 183.

[8] Nayak P.L. Lenka .S and Pati N.C, J. Applied Poly. Science. 25; (1980) 1323

[9] Nayak P.L. Lenka.S and Pati N.C, J. Applied Poly. Science. :26; (1981) 733.

[10] Nayak P.L. Lenka. S, Pradhan A.K, Pati N.C, J. Applied Poly. Science. 19; (1981) 831.

[11] Nayak P.L. Kar S.K and Sahu. G,. Journal of Applied Polymer Science.: 09; (1981) 1521.

[12] Nayak P.L. Kar S.K and Sahu. G. Journal of Applied Polymer Science. :26; (1981) 1413

[13] Nayak P.L. Lenka. S, Pradhan A.K, Mishra M.K: J. Applied Poly. Science: 26; (1981) 2437.

[14] Nayak P.L. Lenka .S, Pradhan A.K, Mishra M.K, J. Applied Poly. Science.: 26; (1981) 2773.

[15] Dash M., Chiellini F., Ottenbrite R.M., Chiellini E.: Chitosan-a versatile semi-synthetic polymer in biomedical applications. J.P. Progress in Polymer Science, 36(2011), 981-1014.

[16] Liu Z., Jiao Y., Wang Y., Zhou C., Zhang Z.: Advanced Drug Delivery Reviews, 60, (2008) 1650-1662.

[17] Alves N.M., Mano J.F.: 43, (2008) 401-414.

[18] Yi H., Wu L.Q,. Bentley W.E., Ghodssi R., Rubloff G.W., Culver J.N., Payne G. F.: Biomacromolecules, 6, (2005) 2881-2894.

[19] Suh J.K., Matthew H.W.: Review. Biomaterials, 21, (2000), 2589-2598.

[20] Jiang X., Cai K., Zhang J., Shen Y., Wang S., Tian X.: Journal of Hazardous Materials, 185, (2011) 1482-1488.

[21] Singh V., Sharma A.K., Tripathi D.N., Sanghi R.: Journal of Hazardous Materials, 161, (2009), 955-966.

[22] Jayakumar R., Prabaharan M., Reis R.L., Mano J.F.: Carbohydrate Polymers, 62, (2005) 142-158.

[23] Bhattacharya A., Misra B.N.: Progress in Polymer Science, 29, (2004) 29767-814.

[24] Prashanth K.V.H., Tharanathan R.N.: Carbohydrate Polymers, 54, (2003) 343-351

[25] Jenkins D.W., Hudson S.M.: Chemical Reviews, 101, (2001) 3245-3273.

[26] Rodrigues, C. A., Laranjeira, M. C. M., Stadler, E., \& Drago, V., Carbohydrate Polymers (2000) 41, 311-314.

[27] Khanal, D. P., Okamoto, Y., Miyatake, K., Shinobu, T., Shigemase, Y., Tokura, S., et al., Carbohydrate Polymers 44, (2001) 99106. 